\title{
Alternate day versus daily atorvastatin in low-density lipoprotein cholesterol reduction: A meta-analysis
}

\author{
Ajoe John Kattoor MBBS, Jawahar L Mehta MD PhD
}

\begin{abstract}
AJ Kattoor, JL Mehta. Alternate day versus daily atorvastatin in low-density lipoprotein cholesterol reduction: A meta-analysis. Curr Res Cardiol 2016;3(3):71-74.
\end{abstract}

Statins are the mainstay treatment for hyperlipidemia. They are hydroxymethylglutaryl-CoA inhibitors and cause reduction in low-density lipoprotein cholesterol (LDL-c) levels. Atorvastatin is one of the most commonly used statins. These drugs are usually prescribed in a daily dose regimen. Due to the long duration of action and prolonged effect on hepatocytes, alternate day atorvastatin therapy is theoretically as effective as daily dose atorvastatin. Several studies have compared the efficacy of alternate day and daily atorvastatin in LDL-c reduction. The authors performed a metaanalysis on these studies to find evidence for alternate day atorvastatin use in LDL-c reduction. The studies comparing alternate day and daily atorvastatin regimens were selected after a literature search. LDL-c reduction in both the alternate and daily groups were calculated from the data provided in the individual studies. The mean difference in LDL-c reduction was compared between the alternate day and daily atorvastatin groups. Metaanalysis performed on the studies revealed that the mean difference in LDL-c reduction among the alternate day and daily groups was only 8.36 $\mathrm{mg} / \mathrm{dL}$ ( $95 \%$ CI -0.49 to 17.20 ). This difference was statistically not significant but trends toward a daily regimen. Further subgroup analysis suggested that the difference in LDL-c reduction is smaller in an atorvastatin-naive patient population (mean difference $0.92 \mathrm{mg} / \mathrm{dL}[95 \%$ CI -13.55 to $15.39 \mathrm{mg} / \mathrm{dL}]$ ) and also in populations with fewer risk factors for cardiovascular disease (mean difference $3.79 \mathrm{mg} / \mathrm{dL}$ (95\% CI -6.40 to $13.98 \mathrm{mg} / \mathrm{dL}])$. In conclusion, the use of alternate day atorvastatin can reduce the cost by one-half and possibly offset many of its side effects. However, long-term studies with large sample sizes are required to evaluate its effect on cardiovascular events and mortality.

Key Words: Alternate day atorvastatin; Alternate day statin; Alternate and daily statin; Atorvastatin and LDL; Non-daily atorvastatin

\section{METHODS}

Statins, hydroxymethylglutaryl (HMG)-CoA inhibitors that block $\checkmark$ cholesterol synthesis, are one of the most commonly used drug classes in cardiovascular medicine today. Their use results in significant reduction in low-density lipoprotein cholesterol (LDL-c) levels. LDL-c is believed to be directly involved in the development of atherosclerosis and has been a primary target in reducing risk for coronary artery disease (CAD) (1). Of the statins available in the market, atorvastatin, rosuvastatin and simvastatin are the most commonly used.

The effect of statins on LDL-c lowering persists several weeks beyond discontinuation of the drug. Atorvastatin, along with its active metabolites, has a half-life of $14 \mathrm{~h}$ to $30 \mathrm{~h}$ (2). Moreover, statins have prolonged action on hepatocytes. The long duration of action of statins along with long residence of LDL-c in plasma (halflife 2.5 days) supports the concept of using statins every other day (3). Several studies have been performed to test the concept of alternate day statin use with atorvastatin. Few studies have been performed with rosuvastatin and simvastatin.

Because statins are to be used long term, use of an alternate day regimen can cut the cost by one-half and, possibly, offset many of their side effects. It is noteworthy that patients worldwide collectively have consumed more than $\$ 100$ billion worth of atorvastatin since its inception approximately 20 years ago.

There have been multiple trials comparing the effectiveness of alternate day versus daily use of statins. However, most of the studies were of small sample size; therefore, it is difficult to make a recommendation based on any single study. We performed a metaanalysis of the studies on alternate day statin use to find evidence for its efficacy in LDL-c reduction. Because most of the studies were performed with atorvastatin, we decided to perform the analysis based on studies that investigated atorvastatin.
A literature search was performed using Pubmed, Google Scholar and the Cochrane library. All studies published until March 1, 2016 were considered for review. Keywords included "alternate day statin", "alternate day atorvastatin". Search for additional studies were performed by cross reference.

Following the literature search, studies that compared alternate day with daily atorvastatin were selected. Inclusion criteria for the study were: studies comparing alternate day versus daily dosing of atorvastatin; and studies published in the English language literature. Studies that involved different atorvastatin dose on alternate day and daily schedules were excluded. For example, the study by Matalka et al (4), which compared alternate day and daily atorvastatin, was excluded. The investigators in this study uptitrated the dose of atorvastatin in alternate day and daily groups to attain the LDL-c goal but the uptitration was not performed equally in both groups and, thus, the data were not comparable. The studies that used a combination of atorvastatin with other lipid-lowering agents were also not included. Studies that showed LDL-c reduction using alternate day or daily dosing without a head-to-head comparison were also excluded.

For comparison, mean difference in LDL-c reduction in each alternate day and daily group was calculated. LDL-c reduction was defined as the mean difference in LDL-c after statin use compared with baseline LDL-c levels.

Review Manager (RevMan version 5.3 [Copenhagen: The Nordic Cochrane Centre, The Cochrane Collaboration, 2014]) was used for meta-analysis. Open Meta-Analyst was also used to aid data retrieval. Preintervention/baseline LDL-c and post intervention LDL-c were

\section{Data collection}

Central Arkansas Veterans Healthcare System, and the Division of Cardiology, University of Arkansas for Medical Sciences, Little Rock, Arkansas, USA Correspondence: Dr JL Mehta, Division of Cardiology, University of Arkansas for Medical Sciences, Little Rock, Arkansas 72212, USA.

Telephone 501-296-1426, e-mail mehtaj@uams.edu 


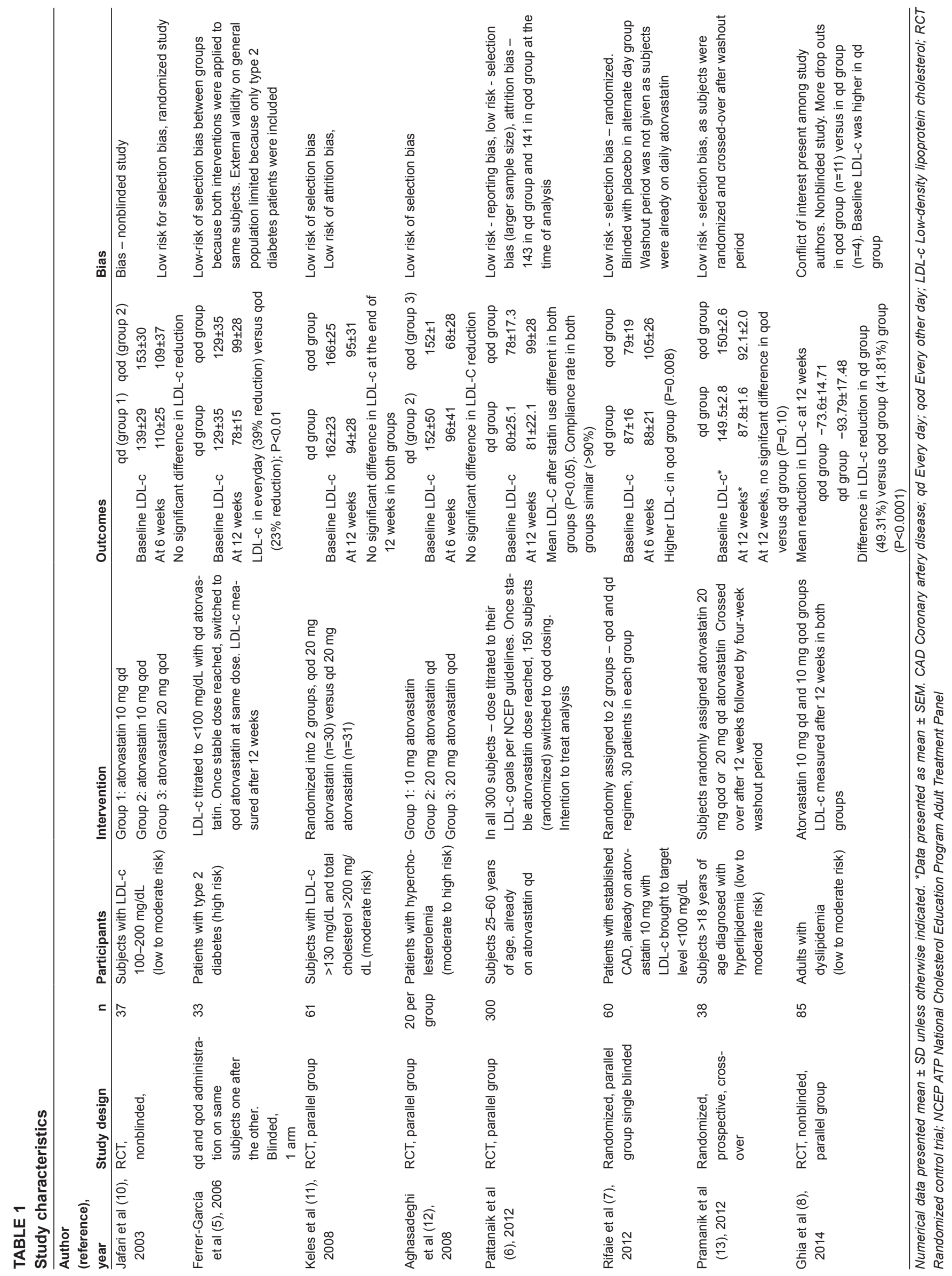




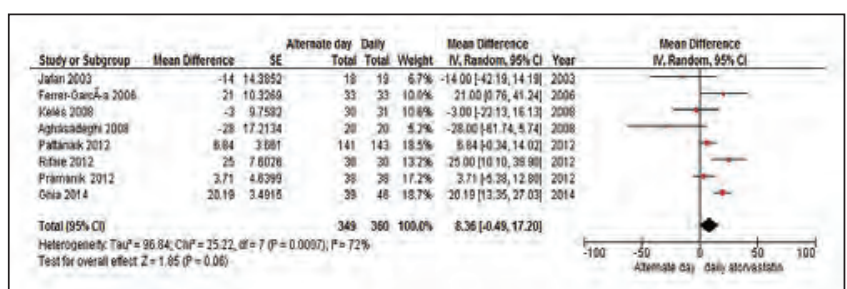

Figure 1) Difference in mean low-density lipoprotein cholesterol reduction in alternate day versus daily statin regimens

obtained from the studies in each alternate day and daily groups. Subsequently, the LDL-c reduction in each group was calculated using Open Meta-Analyst (if not provided directly in the study). The differences in LDL-c reduction were then compared for each of the studies for significance. Mean difference in LDL-c reduction, which was defined as the difference of LDL-c reduction in daily statin group and alternate day statin group, was then calculated. Meta-analysis on the studies were performed using an inverse variance, random-effects model using RevMan 5.3. Risk of bias in the studies was assessed using the authors' judgment and is reported in Table 1 . The summary measure is, hence, the difference in LDL-c reduction between the daily and alternate day atorvastatin groups.

\section{RESULTS}

Initially, 46 studies were selected after narrowing the preliminary search. After inclusion and exclusion criteria were applied, eight studies were selected. Characteristics of the selected studies are summarized in Table 1.

Most participants included in these studies were those who had dyslipidemia but did not have acute coronary syndrome or had undergone percutaneous coronary intervention in the recent past. Patients with serum triglyceride levels $>400 \mathrm{mg} / \mathrm{dL}$, abnormal liver function, hypothyroidism, uncontrolled diabetes, chronic alcoholism, concurrent cholesterol-lowering medications, and use of medications that interact with cholesterol-lowering effect of statins, such as immunosuppressants and antifungal agents, were also excluded in most studies by the investigators.

In the individual studies, subjects were divided into alternate day and daily atorvastatin groups. Change in LDL-c was then calculated at the end of six to 12 weeks of atorvastatin use. In most studies, patients were not on any statin before the start of the study (ie, the patients were atorvastatin naive at the beginning of the study). However, in some studies (see Table 1), patients were initially on atorvastatin daily dosing before they were divided into alternate day and daily atorvastatin groups. Here, the change in LDL-c after intervention was compared with the baseline LDL-c (when the patient was on daily atorvastatin). It was assumed that this change in LDL-c value was contributed only by the change to alternate day dosing.

The mean difference in LDL-c reduction in each study, 95\% Cls and the sample size in alternate day and daily atorvastatin groups are shown in Figure 1. Analysis of the eight studies using a random-effects model showed a mean difference in LDL-c reduction of $8.36 \mathrm{mg} / \mathrm{dL}$ (95\% CI -0.49 to $17.20 \mathrm{mg} / \mathrm{dL}$ ) between alternate day and daily groups, which was not statistically significant (Figure 1). Therefore, it appears that there was no significant difference in LDL-c reduction in alternate versus daily dose regimen. Of note, there was significant heterogeneity within the studies ( $\mathrm{Tau}^{2}=96.84 ; \mathrm{I}^{2}=72 \%$ ).

\section{Subgroup analysis 1}

Ferrer-García et al (5), Pattanaik et al (6) and Rifaie et al (7) studied changes in LDL-c when patients were switched from a daily to an alternate day atorvastatin regimen. The change in LDL-c was used as a marker for the difference in LDL-c reduction among the two groups in these three studies; the difference in LDL-c reduction favoured

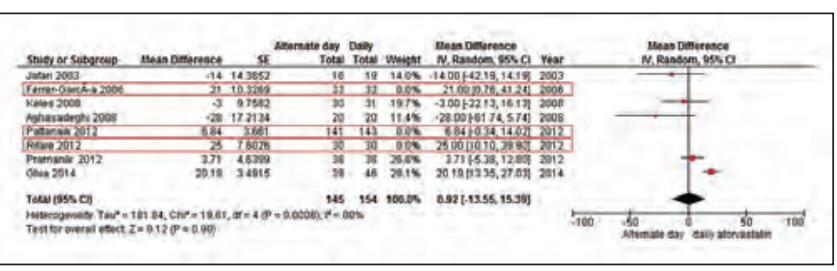

Figure 2) Subgroup analysis 1. Excluding studies in which patients were on daily atorvastatin before study commencement

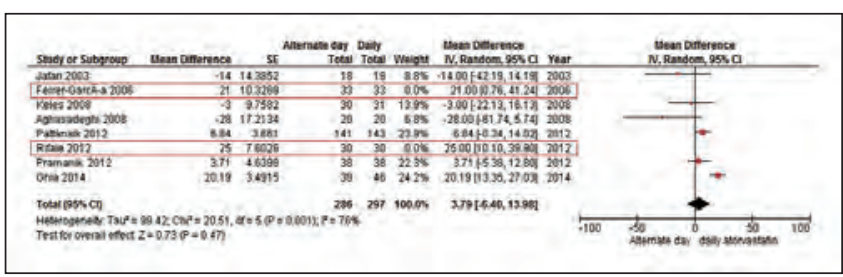

Figure 3) Subgroup analysis 2. Excluding studies in which patients had type 2 diabetes or established coronary artery disease

daily atorvastatin therapy. When meta-analysis was performed after eliminating these three studies, the difference in LDL-c reduction was insignificant (mean difference $0.92 \mathrm{mg} / \mathrm{dL}$ [95\% CI CI -13.55 to $15.39 \mathrm{mg} / \mathrm{dL}]$ ) (Figure 2).

\section{Subgroup analysis 2}

Ferrer-García et al (5) compared alternate day and daily atorvastatin regimen in patients with type 2 diabetes, and Rifaie et al (7) studied subjects with established CAD. Analysis of the remaining six studies showed a smaller difference in LDL-c in the alternate day group versus daily atorvastatin group (mean difference $3.79 \mathrm{mg} / \mathrm{dL}[95 \%$ CI -6.40 to $13.98 \mathrm{mg} / \mathrm{dL}]$ ), which again, was not statistically significant (Figure 3).

\section{DISCUSSION}

Because of the long half-life of atorvastatin metabolites, it has been postulated that alternate day atorvastatin can be effectively used for LDL-c reduction while achieving efficacy similar to daily atorvastatin. Most of the studies comparing alternate day with daily atorvastatin to date had a small sample size. We, therefore, performed meta-analysis to compare the difference in LDL-c reduction between the alternate day and daily atorvastatin regimens.

We included studies that were either randomized or cross-over in design. Study design in all studies was similar, except that a few studies $(5-7)$ were performed in patients whose LDL-c levels were already controlled to target range by daily atorvastatin. Based on the results of our meta-analysis, it appears that the degree of LDL-c reduction is similar with alternate day and daily administration of atorvastatin. Subgroup analysis 1 (Figure 2), after removal of studies in which the LDL-c was controlled initially to target levels with atorvastatin daily dose and then the patients were switched to alternate day therapy, clearly suggests equal efficacy of daily or alternate day regimens in an atorvastatin-naive population. Importantly, significant differences in LDL-c reduction were observed among the groups in which the patients were receiving daily atorvastatin at baseline. Whether this is merely a statistical aberration due to low baseline LDL-c levels or evidence of rebound effect when patients were switched to alternate day regimen requires further investigation.

On exclusion of studies by Ferrer-García et al (5) and Rifaie et al (7), which involved patients with type 2 diabetes mellitus and established CAD, respectively, the mean difference in LDL-c reduction with daily and alternate day regimen was only approximately $4 \mathrm{mg} / \mathrm{dL}$ (95\% CI -6.40 to $13.98 \mathrm{mg} / \mathrm{dL}$ ), which is statistically nonsignificant. This suggests that the difference in LDL-c reduction trends nonsignificantly toward the daily regimen, especially when the subjects carry a small risk for CAD. 
Most of the studies included were free from selection bias beucase they were randomized controlled studies. Some were nonblinded. Possible bias among each study is described in Table 1 . In the study by Ghia et al (8), the baseline LDL level between alternate and daily groups were significantly different. Hence, mean reduction in LDL-c may not be comparable. Furthermore, there was conflict of interest among the authors of the study.

We do not have data regarding outcomes such as cardiovascular morbidity and mortality in alternate day versus daily statin therapy. It may be postulated that because the theoretical basis of alternate day atorvastatin therapy lies in the pharmacokinetics of atorvastatin, alternate day statin therapy should provide similar outcomes as the daily regimen. Further studies in this regard need to be performed.

Approximately $20 \%$ to $30 \%$ reduction in risk for cardiovascular diseases can be achieved by using statin for primary prevention. Current American Cardiovascular Society/American Heart Association guidelines does not recommend targeting an ideal LDL-c level when statins are used for primary prevention of CAD (9). Therefore, small differences in LDL-c reduction may not matter as long as outcomes remain same.

Use of alternate day atorvastatin can reduce the cost by one-half. This becomes particularly significant because patients are typically prescribed these drugs for life. Moreover, many patients on atorvastatin are intolerant to the medication(s), and alternate day therapy may be suitable for such patients (3). Statin side effects, such as myalgia and hyperglycemia, are believed to be lower with alternate day statin therapy (2).

\section{REFERENCES}

1. Reindl EK, Wright BM, Wargo KA. Alternate-day statin therapy for the treatment of hyperlipidemia. Ann Pharmacother 2010;44:1459-70.

2. Marcus FI, Baumgarten AJ, Fritz WL Jr EN. Alternate-day dosing with statins. Am J Med 2013;126:99-104.

3. Keating AJ, Campbell KB, Guyton JR. Intermittent nondaily dosing strategies in patients with previous statin-induced myopathy. Ann Pharmacother 2013;47:398-404.

4. Matalka MS, Ravnan MC, Deedwania PC. Is alternate daily dose of atorvastatin effective in treating patients with hyperlipidemia? The Alternate Day Versus Daily Dosing of Atorvastatin Study (ADDAS). Am Heart J 2002;144:674-7.

5. Ferrer-García JC, Pérez-Silvestre J, Martínez-Mir I, HerreraBallester A. Alternate-day dosing of atorvastatin: Effects in treating type 2 diabetic patients with dyslipidaemia. Acta Diabetol 2006;43:75-8.

6. Pattanaik S, Malhotra S, Sharma YP, Pandhi P. Comparison of alternate-day atorvastatin treatment to daily treatment in maintaining LDL-cholesterol targets in patients with variable coronary risk profile. J Cardiovasc Pharmacol 2012;59:479-84.

7. Rifaie $O$, Zahran A, Nammas W. Alternate-day versus daily atorvastatin in coronary artery disease: A randomized study. Anadolu Kardiyol Derg 2012;12:90-6.
Early studies were concerned with compliance to statin therapy when alternate day regimens was used. This was because of the unusual dosing pattern. Using pill boxes may be recommended to improve compliance. In a study involving 300 subjects, Pattanaik et al (6) reported that compliance rates among daily and alternate day groups were essentially similar.

\section{Limitations}

The present meta-analysis was performed on a varied population, which may be considered to be a limitation of the study. It may even be the considered a strength of the study as diverse patient population simulates real-life everyday practice. As with every meta-analysis, publication bias may have been present in our study. Variable study designs and small sample sizes further contribute to heterogeneity.

\section{CONCLUSION}

In a diverse population requiring statin therapy, alternate day atorvastatin therapy appeared to be similar in efficacy in LDL-c reduction compared with daily atorvastatin therapy. This may be true especially in an atorvastatin treatment-naive population. How alternate day statin therapy affects outcomes, including cardiovascular events and stroke, were not studied. Accordingly, large sample-size, long-term trials involving alternate day and daily statin therapy are needed. Importantly, alternate day therapy may be beneficial in terms of reduction in cost and, possibly, fewer side-effects than daily statin therapy.

DISCLOSURES: The authors have no financial disclosures or conflicts of interest to declare.

8. Ghia CJ, Panda AS, Khobragade LR, Rajesh Kumar JHA, Rambhad GS. Alternate day versus once daily atorvastatin for primary prevention of coronary heart disease in naive patients of dyslipidemia. J Clin Diagnostic Res 2014;8:27-31.

9. Smith SC, Grundy SM. ACC/AHA guideline recommends fixeddose strategies instead of targeted goals to lower blood cholesterol. J Am Coll Cardiol 2014;64:601-12.

10. Jafari M, Ebrahimi R, Ahmadi-Kashani M, Balian H, Bashir M. Efficacy of alternate-day dosing versus daily dosing of atorvastatin. J Cardiovasc Pharmacol Ther 2003;8:123-6.

11. Keles T, Akar Bayram N, Kayhan T, et al. The comparison of the effects of standard $20 \mathrm{mg}$ atorvastatin daily and $20 \mathrm{mg}$ atorvastatin every other day on serum LDL-cholesterol and high sensitive C-reactive protein levels. Anadolu Kardiyol Derg 2008;8:407-12.

12. Aghasadeghi K, Zare D. Efficacy of alternate day dosing of atorvastatin. Cent Eur J Med 2008;3:163-6.

13. Pramanik S, Das AK, Chakrabarty M, Bandyopadhyay SK, Ghosh M, Dalai CK. Efficacy of alternate-day versus everyday dosing of atorvastatin. Indian J Pharmacol 2012;44:362-5. 